\title{
A Class III Histidine Kinase Acts as a Novel Virulence Factor in Botrytis cinerea
}

\author{
Muriel Viaud, ${ }^{1}$ Sabine Fillinger, ${ }^{2}$ Weiwei Liu, ${ }^{2}$ Jai Santosh Polepalli, ${ }^{3}$ Pascal Le Pêcheur, ${ }^{1}$ \\ Aditya Reddy Kunduru, ${ }^{3}$ Pierre Leroux, ${ }^{2}$ and Laurent Legendre ${ }^{3}$ \\ ${ }^{1}$ Unité de Phytopathologie et Méthodologies de la Détection, Institut National de la Recherche Agronomique, Rte de St-Cyr, \\ 78026 Versailles, France; ${ }^{2}$ Unité de Phytopharmacie \& Médiateurs Chimiques, Institut National de la Recherche \\ Agronomique, Rte de St-Cyr, 78026 Versailles, France; ${ }^{3}$ Post-Harvest Center for Horticulture and Plant Sciences, \\ University of Western Sydney, Hawkesbury campus, Australia
}

Submitted 10 January 2006. Accepted 16 May 2006.

\begin{abstract}
Filamentous ascomycetes contain large numbers of histidine kinases (HK) that belong to eleven classes. Members of class III from different species were previously shown to be involved in osmoregulation and resistance to dicarboximide and phenylpyrrole fungicides. We have inactivated the gene encoding the single group III HK, BOS1, in the economically important plant pathogen Botrytis cinerea. BOS1 inactivation had pleiotropic effects on the fungus. Besides the expected osmosensitivity and resistance to fungicides, null mutants presented additional characteristics indicating that BOS1 is necessary for normal macroconidiation and full virulence. On standard culture media, null mutants very rarely formed conidiophores and those few conidiophores failed to produce conidia. This defect could be partially restored with $1 \mathrm{M}$ sorbitol, suggesting that another BOS1-independent signal cascade may be involved in macroconidiation. The mutants were not found to be hypersensitive to various oxidative stresses but were more resistant to menadione. Finally, pathogenicity tests showed that bos 1 -null mutants were significantly reduced in the ability to infect host plants. Appressorium morphogenesis was not altered; however, in planta growth was severely reduced. To our knowledge, this is the first class III HK characterized as a pathogenicity factor in a plant-pathogenic ascomycete.
\end{abstract}

Two-component histidine kinase (HK) phosphorelay protein complexes are key components of the signal-sensing machinery of bacteria, yeasts, plants, and fungi. They allow these organisms to adapt to changing environmental conditions by mediating functions as diverse as host recognition, virulence, stress and hormonal response, chemotaxis, quorum-sensing, and osmosensing (Chang and Stewart 1998; Hoch 2000; Wolanin et al. 2003). These phosphorelays contain a HK component that autophosphorylates a conserved histidine residue in response to an external signal. The phosphate is then transferred to a conserved aspartic residue of a receiver domain of a

Corresponding author: M. Viaud: viaud@ versailles.inra.fr

Current address for L. Legendre: Université Jean Monnet de St Etienne, Laboratory of Plant Biotechnology applied to aromatic and medicinal plants, 23 rue du Dr Paul Michelon, 42023 St Etienne Cedex 2, France.

This article is in the public domain and not copyrightable. It may be freely reprinted with customary crediting of the source. The American Phytopathological Society, 2006. response regulator (RR) component. In eukaryotes, two-component systems initiate intracellular signaling pathways that make use of diverse signaling elements such as mitogen-activated protein kinases (MAPKs) and cyclic nucleotides (West and Stock 2001). Among the best-studied signaling cascades is the HOG cascade that mediates the osmotic and oxidative stress responses of the budding and fission yeasts (Hohmann 2002). In Saccharomyces cerevisiae, hyperosmotic conditions lead to the dephosphorylation of a transmembrane HK $(\mathrm{S} \ln 1)$ that negatively regulates a MAPK cascade via the phosphorelay elements Ypd1 and Ssk1. The MAPK cascade functions via the sequential phosphorylation of a MAPKKK (Ssk2/Ssk22), a MAPKK (Pbs2), and the MAPK Hog1, which, upon phosphorylation, migrates to the nucleus to modulate the activity of several transcription factors mediating the downstream transcriptional response. Regulation of the HOG pathway also involves another osmosensing branch, the Sho1 branch, which is functionally partially redundant with the $\operatorname{Sin} 1$ branch and has several protein kinases and phosphatases in common with other MAPK pathways. This offers several opportunities for cross-talk between signaling cascades regulating apparently different cellular functions, such as adaptation to medium osmolarity, cell integrity control, sterile vegetative growth, resistance to elevated temperatures, and oxidative stress (AlonsoMonge et al. 2001; Cullen et al. 2000; Gustin et al. 1998; Lee and Elion 1999). In fission yeast, Sty1, the Hog 1 homolog, mediates cell-cycle control, mating, osmoadaptation, and response to a multitude of stress conditions including heat stress, oxidative stress, exposure to $\mathrm{UV}$, extremes of $\mathrm{pH}$, and starvation (Gustin et al. 1998; Hohmann 2002).

In filamentous fungi, homologs of individual elements of the budding yeast HOG1 and fission yeast Sty1 pathway have been identified by functional and in silico genetics (Dixon et al. 1999; Han and Prade 2002; Kawasaki et al. 2002; Kojima et al. 2004; Krantz et al. 2006a and b; Park et al. 2004; Zhang et al. 2002). Neurospora crassa OS-2 is homologous to budding yeast HOG1 (Zhang et al. 2002) and is regulated by the MAPKK OS-5 and the MAPKKK OS-4 (Fujimura et al. 2003). Potential orthologs of Sln1, Sho1, Ypd1, Ssk1, and Ste11 can be found in the genome sequence of $N$. crassa. However, the phosphorelay mainly responsible for osmosensing, OS-1 (also known as NIK1), is not an ortholog of Sln1 (Alex et al. 1996; Catlett et al. 2003). A whole-genome search for two-component signal transduction genes in the saprophytic fungus $N$. crassa and the phytopathogenic fungi Cochliobolus heterostrophus, Gibberella moniliformis, and Botrytis cinerea (Catlett et al. 2003) revealed the existence of an exten- 
sive family of HK genes that can be grouped into eleven classes. Among them, it is the NIK1/OS-1 class III group (which has representatives in all the four studied euascomycete species) that contains the putative osmosensors. These NIK1/OS-1 class III group polypeptides differ significantly from members of the $\operatorname{Sin} 1 / \mathrm{TcsB} \mathrm{HK}$ class in that, in addition to the usual phosphoacceptor, ATP-binding, and RR domains, they have an Nterminal region that contains several repeats of a HAMP domain, a domain of currently unknown function. They also lack transmembrane domains present in the HK of the SIn 1 class. Genetic studies on NIK1 orthologs in N. crassa, C. heterostrophus, and Magnaporthe grisea showed that null mutants are sensitive to osmotic stress and resistant to the dicarboximide and phenylpyrrole classes of fungicides (Motoyama et al. 2005a; Ochiai et al. 2001; Yoshimi et al. 2004). N. crassa OS-1 has also been shown to be required for the production of asexual reproductive structures (most strikingly under hyperosmotic conditions). More recently, Motoyama and associates (2005b) demonstrated that heterologous expression of the $M$. grisea OS-1/HIK ortholog Hik1 in Saccharomyces cerevisiae yields a functional polypeptide with HK enzymatic activity and the ability to induce phosphorylation of HOG1 in response to the addition of phenylpyrroles, dicarboximides, and aromatic hydrocarbon fungicides. Furthermore, expression of M. grisea Hik1 in $\mathrm{S}$. cerevisiae was found to confer fungicide sensitivity.

The necrotrophic fungus B. cinerea (Botryotinia fuckeliana) is the causal agent of gray mold on a large number of economically important food and ornamental crops (Elad et al. 2004). This fungus is capable of causing diseases on a wide range of plants and is considered to be the most serious microbial threat to ripe fruits including, grapes and strawberries (Mac Clellan and Hewitt 1973). Dicarboximide fungicides have been extensively used to control $B$. cinerea, leading to the widespread occurrence of field-resistant strains (Leroux et al. 2002). Genetic analysis of these mutants has demonstrated that resistance is conferred by a single locus, designated Dafl (Faretra and Pollastro 1991). Recently, Cui and associates (2002) identified the $B$. cinerea BOS1 gene encoding an osmosensing HK NIK1 ortholog and showed that this gene corresponds to the Dafl locus. Moreover, single-point mutations in BOS1 resulting in amino-acid changes were shown to confer both dicarboximide resistance and osmosensitivity. The ability to osmoregulate is likely to be a key determinant of the success of the pathogen within many plant-fungus interactions. For example, in some species, high intracellular osmotic pressure is needed inside the appressorial infection structure to allow penetration into the host plant (de Jong et al. 1997; Howard and Valent 1996). Moreover, many B. cinerea host tissues such as ripe fruit have high internal osmolarity that is likely to inflict severe osmotic stresses on the pathogen. As far as we are aware, the putative role of BOS1 signaling pathway in $B$. cinerea infectious process was not yet investigated. Therefore, we constructed bos 1 specific knock-out mutants in order to evaluate the putative function of BOS1 in virulence. Their phenotypes confirmed that BOS1 is, indeed, involved in osmoregulation and dicarboximide resistance and revealed that functional BOS1 is necessary for macroconidiation and full virulence.

\section{RESULTS}

\section{Inactivation of the BOS1 gene.}

Catlett and associates (2003) performed a whole-genome analysis of HK-encoding genes in $B$. cinerea and identified BOS1 as the unique representative of group III HK that are mainly known as osmosensors. In order to study the biological function of BOS1, we carried out a one-step gene inactivation as shown in Figure 1. The knock-out plasmid, pBOS1D, was constructed, using a polymerase chain reaction (PCR)-based strategy (discussed below), and harbors the $B A R$ gene conferring resistance to bialaphos in place of the central part (from aa 192 to 908) of the BOS1 gene (Cui et al. 2002). By homologous recombination at the BOS1 locus, this plasmid is expected to remove HAMP, HK, and ATPase domains. pBOS1 1 was linearized and used to transform protoplasts of the UWS111 wild-type (WT) strain. Regeneration and further purification on bialaphos selective medium led to the isolation of 75 transformants. Among the 75 transformants, 45 formed fast-growing colonies after 5 days of culture on bialaphos medium containing $2 \mu \mathrm{g}$ of iprodione per milliliter (this we termed dicarboximide resistant). In contrast, the UWS111 WT strain and the other 30 transformants were completely unable to grow on this medium and were characterized as sensitive to the fungicide. PCR amplifications with one primer located upstream of the $B O S 1$ gene and one primer located inside the BAR gene (discussed below) confirmed that the expected gene replacement event had taken place in dicarboximide-resistant transformants (data not shown). Two sensitive transformants (32 and 35) and two resistant transformants (36 and 72) were selected and ana-
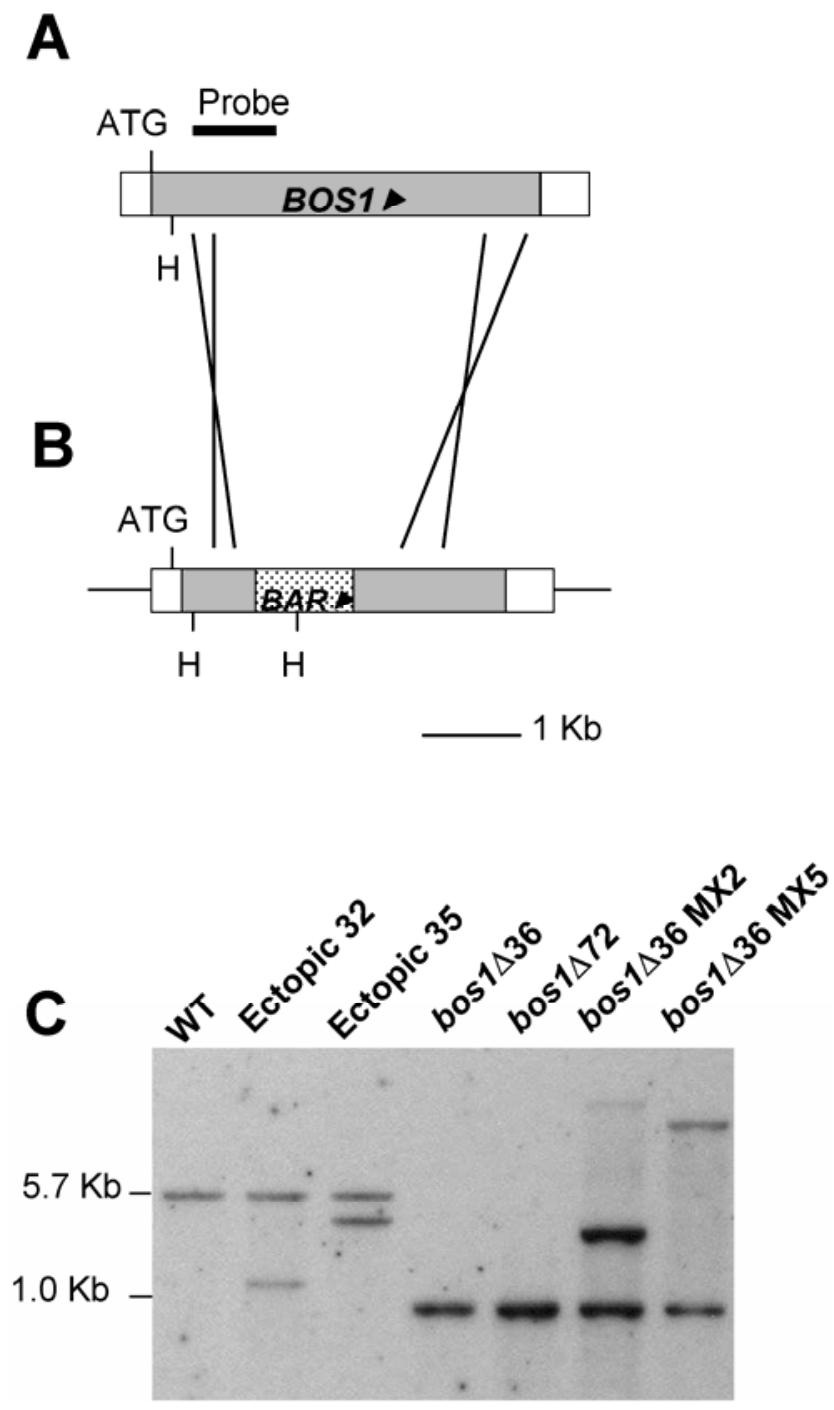

Fig. 1. BOS1 gene inactivation by homologous recombination. A, Schematic representation of the BOS1 locus. $\mathrm{H}$ indicates HindIII restriction site. B, $\mathrm{p} B O S 1 \triangle$ knock-out vector. $\mathbf{C}$, Southern blot showing the molecular patterns of UWS111 wild-type (WT) strain, ectopic transformants 32 and 35 , bos $1 \Delta$ null mutants 36 and 72, and complemented mutant bos $1 \Delta 36 \mathrm{MX} 2$ and MX5. DNA was digested with HindIII, and the blot was probed as indicated in A. 
lyzed by Southern blot. Hybridization patterns confirmed that transformants 36 and 72 (hereafter named bos $1 \Delta 36$ and bos 1 $\triangle 72$ ) were the result of a single homologous recombination event at the BOS1 locus, while transformants 32 and 35 were the result of single ectopic integrations (Fig. 1C).

In order to validate gene inactivation phenotypes, we introduced a BOS 1 WT copy at an ectopic locus into the bos $1 \triangle 36$ null mutant using the plasmid pSF06 (discussed below). After isolating 20 transformants, we selected two, bos $1 \Delta 36 \mathrm{MX} 2$ and bos $1 \Delta 36 \mathrm{MX} 5$ (Fig. 1C), as controls for all further experiments. bos $1 \Delta 36 \mathrm{MX} 5$ is a single-copy transformant, whereas Southern blotting suggested that bos $1 \Delta 36 \mathrm{MX} 2$ carries two copies of the BOS1 WT gene.

\section{BOS1 is involved in fungicide resistance.}

Resistance of bos1-null mutants to the dicarboximide iprodione and the phenylpyrrole fludioxonil was tested as shown in Figure 2 (upper lane). Both bos $1 \Delta 36$ and bos $1 \Delta 72$ mutants grew on high concentrations of iprodione $\left(25 \mu \mathrm{g} \mathrm{ml}^{-1}\right)$ and fludioxonil $\left(1 \mu \mathrm{g} \mathrm{ml}^{-1}\right)$, whereas the two complemented mutants exhibited the same sensitive phenotype as the parental
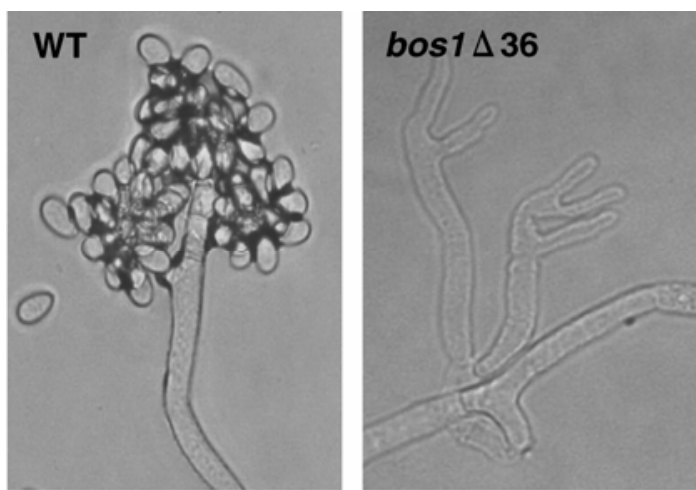

Fig. 3. Conidiophore formation and conidiation. Aerial hyphae from UWS111 wild-type strain and bos $1 \Delta 36$ null mutant were detached from one-week-old mycelium (grown on nutrient yeast medium) using transparent adhesive tape, which was then placed onto a glass slide and viewed using a light-transmission microscope.

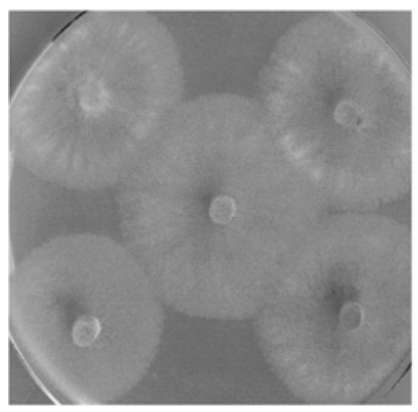

Control

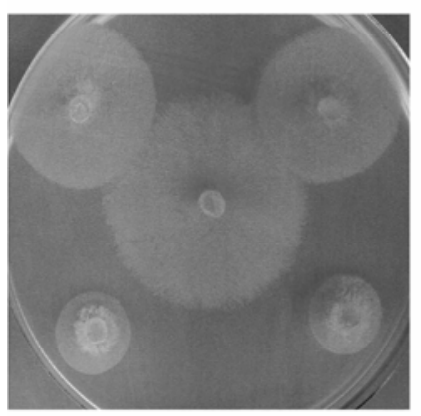

$\mathrm{NaCl} 0.3 \mathrm{M}$

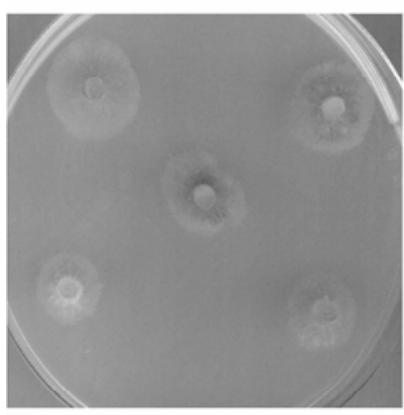

$\mathrm{H}_{2} \mathrm{O}_{2} 24 \mathrm{mM}$

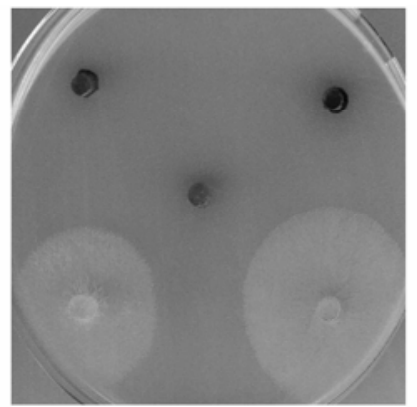

Iprodione $25 \mu \mathrm{g} \mathrm{ml}^{-1}$

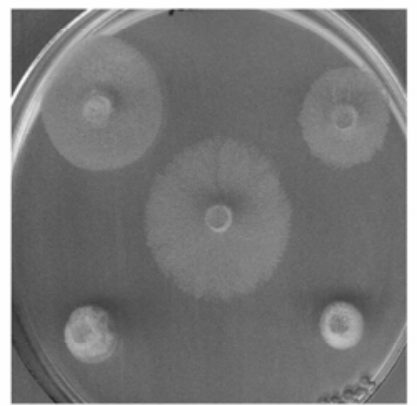

$\mathrm{NaCl} 0.6 \mathrm{M}$

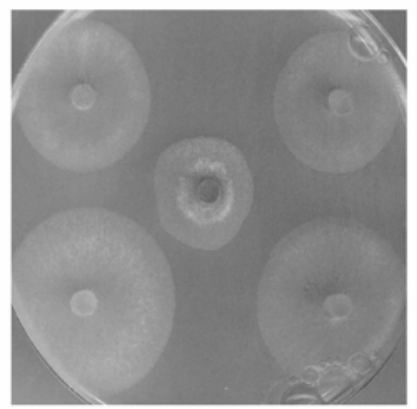

Menadione $250 \mu \mathrm{M}$

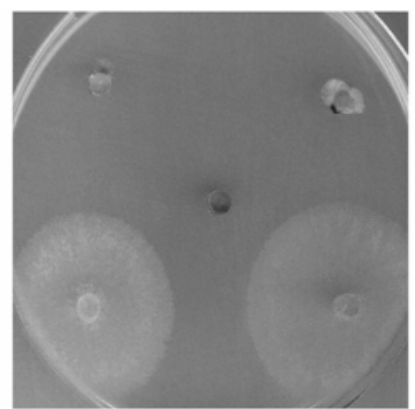

Fludioxonil $1 \mu \mathrm{g} \mathrm{ml}^{-1}$

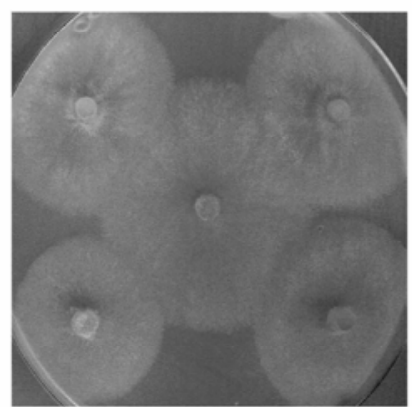

Sorbitol $0.05 \mathrm{M}$

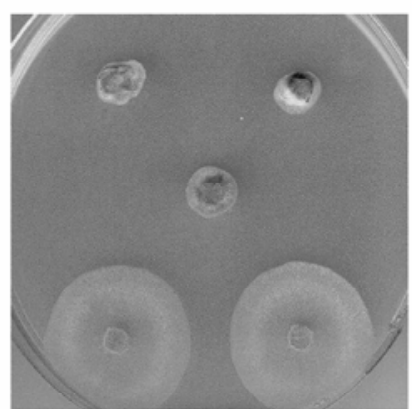

Menadione $500 \mu \mathrm{M}$

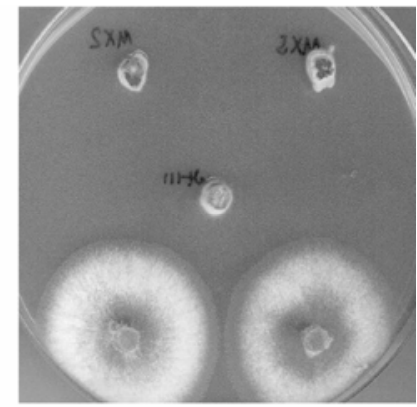

Quintozene $25 \mu \mathrm{g} \mathrm{ml}^{-1}$

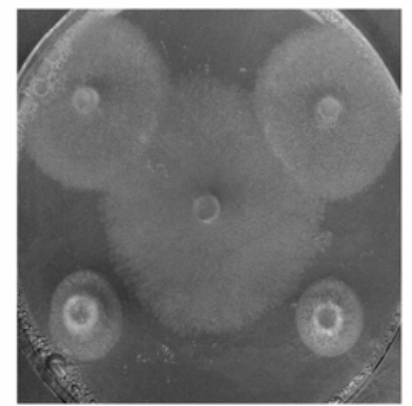

Sorbitol $0.5 \mathrm{M}$

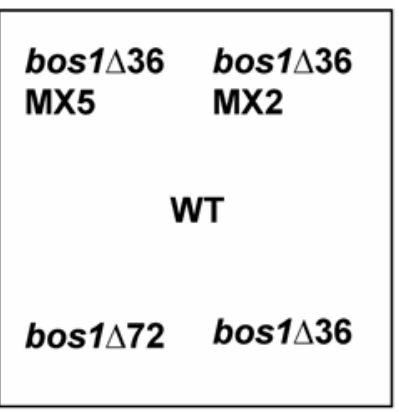

bos1 $\Delta 72 \quad \operatorname{bos} 1 \Delta 36$

Fig. 2. Sensitivity to fungicides, osmotic, and oxidative stresses. In vitro growth tests of bos $1 \Delta$ null mutants (36 and 72 ) and complemented mutants (bos1 $\triangle 36 \mathrm{MX} 2$ and MX5) were carried out on nutrient yeast medium supplemented as indicated; upper lane, fungicides; middle lane, osmotic stress; and lower lane, oxidative stress. Mycelial plugs were placed on the plates as indicated in the key in the bottom right hand corner. Growth was scored after 2 to 3 days of incubation at $21^{\circ} \mathrm{C}$. 
WT strain UWS111. Both mutants were also resistant to the aromatic hydrocarbon fungicide quintozene, as described previously for dicarboximide-resistant laboratory isolates (data not shown) (Cui et al. 2002; Leroux et al. 2002). No resistance to other families of fungicides (antimicrotubules, anilinopyrimidines, hydroxyanilides) was detected in any of the mutants (data not shown). Therefore, a functional BOS1 HK mediates resistance to three types of fungicides, dicarboximides, phenylpyrroles, and aromatic hydrocarbons.

\section{BOS1 is necessary for normal osmoregulation.}

The osmosensitivity of bosl-null mutants was estimated on media supplemented with different concentrations of sodium chloride, glucose, or sorbitol. As shown in Figure 2 (middle lane), bos $1 \Delta 36$ and bos $1 \Delta 72$ null mutants exhibited reduced radial growth in comparison with the WT strain and complemented mutants on all hyperosmotic media. Under conditions of low-sugar concentrations (0.05 M sorbitol or glucose), growth was comparable to the parental strain. These results indicate that a functional BOS1 gene is necessary for the adaptation to a hyperosmotic environment.
BOS1 is not the general oxidative stress signal transducer.

In order to know whether BOS1 HK is involved in oxidative stress responses, growth of bosl-null mutants, WT strain, and complemented mutants was tested on nutrient yeast (NY) medium supplemented with oxidative stress inducers $\left(\mathrm{H}_{2} \mathrm{O}_{2}\right.$, paraquat, $\mathrm{Cu}^{2+}, \mathrm{Cd}^{2+}, \mathrm{Zn}^{2+}$, menadione). None of these conditions resulted in differential growth of the null mutants and control strains (Fig. 2, lower lane; data not shown). These results suggest that BOS1 inactivation does not lead to increased sensitivity to oxidative stress. Surprisingly, however, we did observe that bosl mutants showed an increased resistance to menadione, which has been reported to induce oxidative stress.

\section{Normal asexual development is dependant on BOS1.}

When grown on standard culture media (NY or potato dextrose agar [PDA]), in contrast to the WT strain and the complemented mutants, bos 1 -null mutants $\Delta 36$ and $\Delta 72$ did not produce any macroconidia. Macroconidiation was tested on other media containing bean-leaf extract or tomato juice, and we, again, observed a failure to form macroconidia in bosl-null mutants (data not shown). Black light, which is known to in-

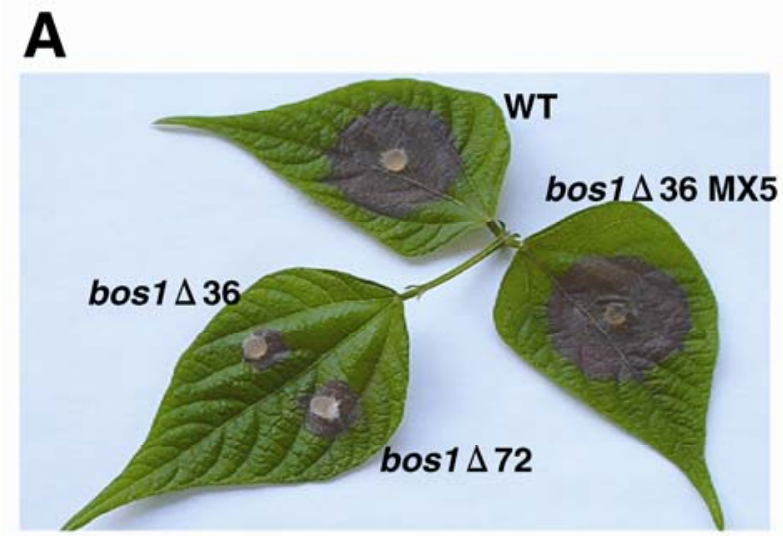

C

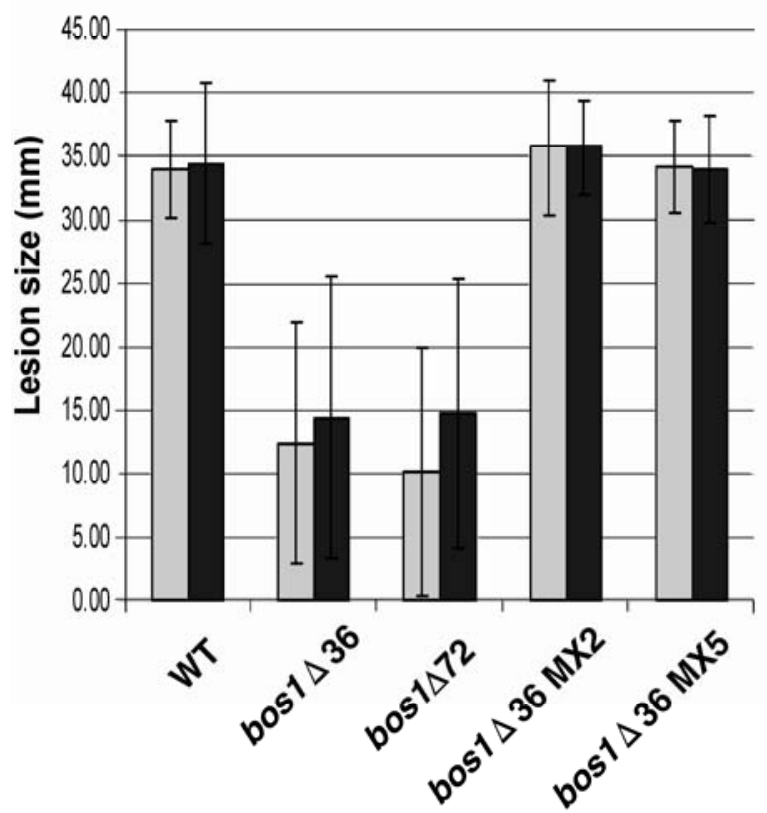

B
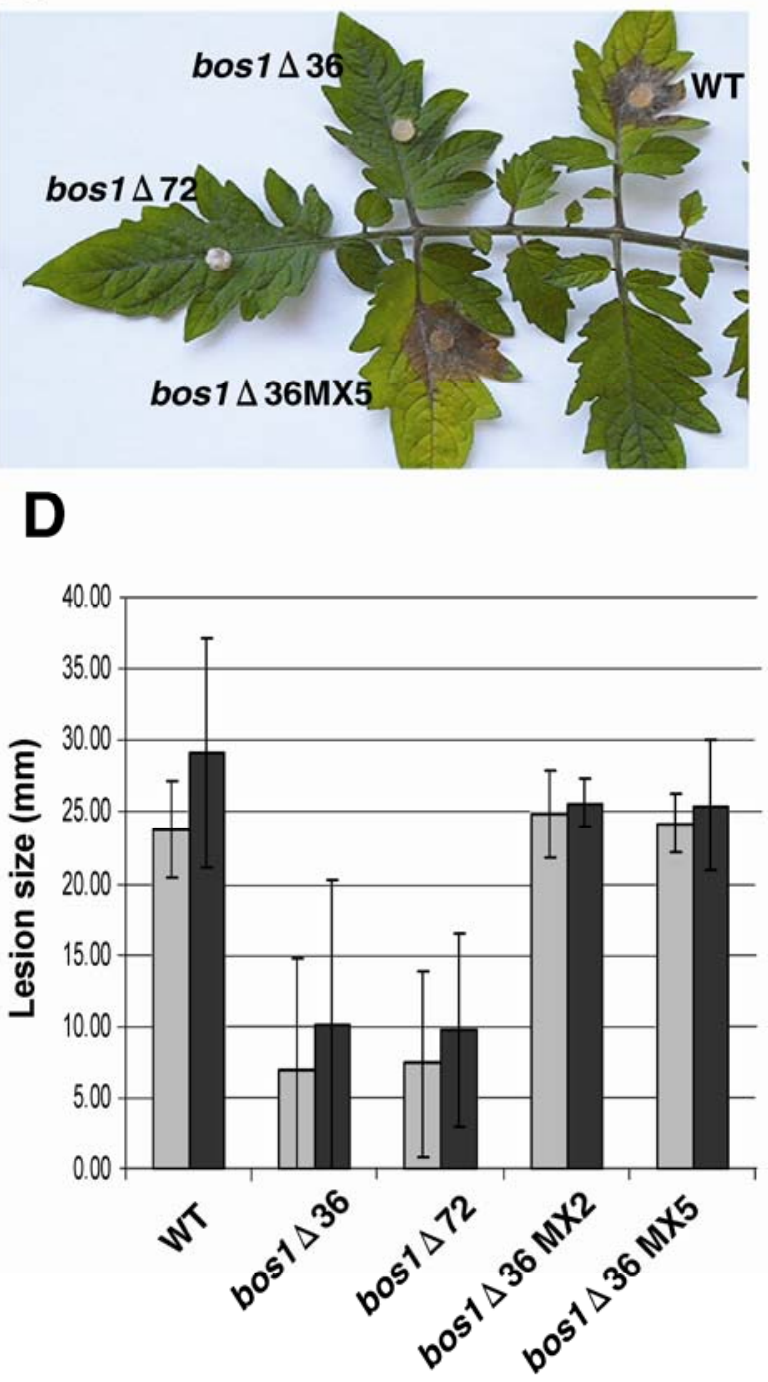

Fig. 4. Pathogenicity assays on $\mathbf{A}$ and $\mathbf{C}$. bean and $\mathbf{B}$ and D, tomato. Leaves were inoculated with plugs of mycelium from UWS111 wild-type strain, bos $1 \Delta$ null mutants (36 and 72), and complemented mutants (bos1 136 MX2 and MX5). A and B, Pictures of intact leaves were taken 2 days postinfection (dpi). C and $\mathbf{D}$, Histograms representing average lesion diameters of the above-described strains on intact (black columns) or wounded (gray columns) leaves 3 dpi. The bars indicate standard deviations of three independent assays with five to seven independent leaves per replicate. 
crease fungal conidiation, was also ineffective on bos 1-mutant conidiation (data not shown). Microscopic observations showed that bos1-null mutants are reduced in aerial hyphal development and in conidiophore formation. Only a few abnormal conidiophores completely devoid of conidia could be observed (Fig. 3). The bos 1 -null mutants, however, still produced conidia when grown on plants. Moreover, a medium containing $1 \mathrm{M}$ sorbitol was able to rescue the macroconidiation defect of null mutants. Indeed, the bos 1 -null mutants produced macroconidia at about $10 \%$ of WT level on this medium (data not shown). In contrast to their macroconidiation defect, bos 1 null mutants were still able to produce microconidia and sclerotia (data not shown). These results indicate that BOS1 is necessary for conidiation under standard in vitro conditions.

\section{BOS1 is required for normal virulence.}

The involvement of BOS1 in pathogenicity was first evaluated with standard bean and tomato tests using mycelial plugs (Fig. 4). Both bos 1 -null mutants were significantly less virulent than the WT strain on both host plants. At 3 days postinfection (dpi), both mutants produced lesions with average lesion diameters representing 29 to $43 \%$ of WT values. Further monitoring of the progress of infection showed that this reduction in virulence was still significant after 10 days (data not shown). A similar reduction in virulence was observed on grape berries and apples (data not shown; Fig. 5). Figure 4 shows that complemented mutants were fully pathogenic, thereby confirming that the reduction in virulence was caused by the inactivation of the BOS1 gene.

In order to test whether the reduction in virulence of the bos 1-null mutant was related to a defect in penetration of the leaf cuticle, bean and tomato leaves were wounded prior to inoculation. This treatment did not accelerate infection by the null mutants (Fig. 4). Furthermore, microscopic observation of penetration-related morphogenesis on onion epidermis revealed that bos 1-null mutants developed appressorial infection structures and internal infection hyphae that were similar to those of the original WT strain (data not shown). At $18 \mathrm{~h}$ after inoculation, $81.1 \pm 8.5 \%$ of the WT conidia had differentiated an appressorium directing the penetration of the fungus into the underlying epidermal cell, which is similar to the results of Gourgues and associates (2004). This percentage was not significantly different in the bosl-null mutant $(75.9 \pm 9.0 \%)$. Taken together, these results suggest that BOS1 may be required in the postpenetration stages of the infection process on several hosts and organs (leaves and fruits).

\section{DISCUSSION}

\section{Stress response.}

The present study of B. cinerea BOS 1 class III HK confirms previous studies that have suggested that this type of protein is

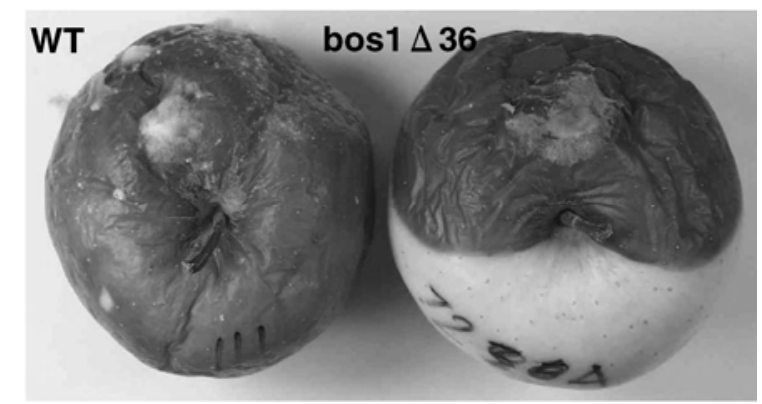

Fig. 5. Pathogenicity assays on apples. Fruits were inoculated with plugs of mycelium from UWS111 wild-type (WT) strain and bos $1 \Delta 72$ null mutant. involved in resistance to three classes of fungicides, dicarboximides, phenylpyrroles, and aromatic hydrocarbons. It is suspected that these fungicides may share a common mode of action, as they all affect germination, mycelial outgrowth through DNA synthesis, and the progress of the cell cycle (Leroux 2004; Leroux and Fritz 1984). Exactly how BOS1 inactivation confers resistance to these fungicides remains to be elucidated.

The phenotype of the bos 1-null mutants suggests that $B$. cinerea BOS1 HK is involved in the osmotic-stress response in this species, as has been reported for the corresponding orthologous gene products in $N$. crassa, M. grisea, and C. heterostrophus (Motoyama et al. 2005a; Schumacher et al. 1997; Yoshimi et al. 2004). Indeed, growth of bos1-null mutants was inhibited by both high salt and high sugar concentrations. Nevertheless, in contrast to the yeasts $S$. cerevisiae and Schizosaccharomyces pombe, gene inactivation of the HK osmosensor protein BOS1 in $B$. cinerea did not affect its sensitivity to oxidative stress. Interestingly, BOS1 gene inactivation induced increased resistance to menadione. This compound has been shown to induce oxidative stress by redox cycling in hepatocytes (Di Monte et al. 1984a and b). Tzeng and associates (1995) could show menadione-mediated toxicity through induction of lipid peroxidation in cardiocytes. In fungi, dicarboximide treatment is also known to lead to lipid peroxidation (Choi et al. 1996; 1997). In light of these data, one explanation for the cross-resistance of bos 1 -null mutants to dicarboximides and menadione could be differences in the degree of lipid peroxidation in between the mutants and the WT strain. Testing this hypothesis experimentally could shed some light on the origin of this cross-resistance to dicarboximide and menadione.

\section{Development.}

Inactivation of BOS1 led to a severe reduction in the development of aerial hyphae and conidiophores that completely blocked the formation of macroconidia under standard axenic culture conditions. Similar results were observed when a member of group IV of the HK (TcsA) gene was deleted in Aspergillus nidulans (Virginia et al. 2000). According to Catlett and associates (2003), B. cinerea and C. heterostrophus do not contain any group IV HK. These authors suggest that the unequal distribution of this group of $\mathrm{HK}$ across fungal species correlates with different modes of development of macroconidia. Aspergillus species produce macroconidia enteroblastically from phialides, whereas Botrytis and Cochliobolus species form macroconidia holoblastically. In contrast to macroconidia, the production of microconidia in $B$. cinerea occurs enteroblastically from phialides and is not affected by BOS1 inactivation. These observations suggest that $\mathrm{BOS} 1$ is involved in holoblastic conidiation.

It is noteworthy that bos 1-null mutants still produce macroconidia when developing on plants or on $1 \mathrm{M}$ sorbitol medium. Similar results were observed for Aspergillus nidulans, in which the conidiation defect in the $\operatorname{tcs} A$ null mutant was also suppressed by growth on $1 \mathrm{M}$ sorbitol (Virginia et al. 2000). These results indicate that BOS1-independent signaling cascades may play a role in conidiation under specific physiological conditions, such as in planta growth or hyperosmotic conditions.

In yeast, two upstream signaling cascade branches regulate Hog1 activity, the Sho1-dependent and the Sln1-dependent branch. The functions of these branches are redundant on highosmolarity medium; however, the phosphorylation of Hog 1 at low-solute concentrations on one hand and at very high-solute levels on the other is more dependent on the SIn1 branch (Maeda et al. 1995; O'Rourke et al. 2002; van Wuytswinkel et al. 2000). Additional osmolarity-sensing inputs have been reported that interact with the Sho1 branch (O'Rourke et al. 
1998; van Wuytswinkel et al. 2000). In a very recent wholegenome analysis of the HOG pathway in 20 published fungal genomes, Krantz and coworkers (2006a and b) showed that homologs of Sho1 are present in nearly all the fungal species analyzed. We were, however, unable to identify a Sho1 ortholog in the $B$. cinerea genome (Botrytis cinerea web database) with blast searches (data not shown), whereas a $\operatorname{Sln} 1$ ortholog named BfHHK5 has previously been identified (Catlett et al. 2003). Whether BfHHK5 or another signaling branch is involved in macroconidiation restoration on high osmolarity medium (1 M sorbitol) and whether this regulation is mediated through phosphorylation of Hog1-related MAPK remains to be investigated.

\section{Pathogenicity and fitness.}

Our phenotypic characterization of $B$. cinerea bos 1 -null mutants showed that a class III HK is an important virulence determinant in this fungus. As far as we are aware, this is the first report of HK enzyme acting as a virulence factor in a phytopathogenic fungus. In the absence of BOS1, the extent of lesion development was significantly reduced on all hosts tested. Microscopic observations showed that appressorium differentiation and penetration of onion epidermis still occur in bos 1-null mutants, indicating that BOS1 is not essential for these morphogenetic processes. Moreover, artificial wounding of plant leaves did not restore the full virulence of the boslnull mutants, further suggesting that a penetration deficiency is not the cause of the reduced lesion development in these mutants. These data are consistent with a role for BOS1 in the postpenetration stages of the infection process that allow the colonization of the host. One hypothesis that could be considered is that bos 1 mutants develop more slowly in the hosts tested because of stresses imposed by the intrinsic osmotic state of these hosts. In the cereal pathogen $M$. grisea, inactivation of a homologous HK gene did not impair pathogenicity (Motoyama et al. 2005a). However, other phenotypic aspects, including resistance to the same three groups of fungicides, osmosensitivity, and the formation of functional appressoria, are shared by the $\Delta h i k 1$ mutant of $M$. grisea and the B. cinerea bos 1-null mutants. In Alternaria brassicicola, another phytopathogenic fungus, frameshift and nonsense mutations in the AbNIK1 NK-encoding gene have been identified in field isolates cross-resistant to iprodione and fludioxonil (Avenot et al. 2005). In a similar manner to the $M$. grisea $\Delta$ hikl mutants, these $\triangle A b n i k 1$ mutants are also osmosensitive but not impaired in virulence. Taken together, these data suggest that class III HK may play different roles in the infectious processes of the three above-cited phytopathogenic fungi. This may relate to the fact that these species belong to different phyla (B. cinerea is a leotiomycete, M. grisea is a sordariomycete, and Alternaria brassicola is a dothideomycete) and that they differ in their infection processes and in host range; $B$. cinerea is a necrotrophic and polyphageous pathogen of different organs including leaves and fruits, while $M$. grisea is a hemibiotrophic pathogen of cereal leaves, and Alternaria brassicola is a seedborne necrotrophic pathogen of Brassicaceae. The differential functions of the class III HK in these pathosystems may be linked to different fungal strategies for the management of host osmolarity during plant colonization or to differences in the level of osmotic stress encountered (difference between the hosts). Alternatively, the inactivation of $B$. cinerea BOS1 might impinge on other signaling cascades and result in uncoordinated activity of various other pathogenicity-related cellular functions in this fungus. More functional data on these and additional species need to be obtained to elucidate the phylogenetic origin, the biological implications, or both of these divergent HK functions.
The involvement of the $B$. cinerea BOS1 HK in the infection process and macroconidiation may explain the absence of dicarboximide-phenylpyrrole cross-resistant strains in nature despite the selection pressure of alternate applications of both fungicides on several crops (Leroux et al. 2002; Moyano et al. 2004). These cross-resistant strains would be predicted to exhibit reduced fitness in the field, since they have a reduced ability to reproduce asexually (asexual reproduction is considered the main mode of dispersion for $B$. cinerea) and their growth in the plant hosts is also reduced. Raposo and associates (2000) have also reported that $B$. cinerea dicarboximideresistant sclerotia have a lower survival rate during overwintering, which would place a further limit on the dispersal of cross-resistant strains. In a very recent study, Ziogas and coworkers (2005) evaluated various different fitness parameters of in vitro-generated phenylpyrrol resistant $B$. cinerea mutants that fell into two classes, omosensitive and osmoresistant. All osmosensitive isolates showed significant reductions in their saprophytic fitness characteristics, including mycelial growth, sporulation, conidial germination, and sclerotia production. Studies on the competitive ability of mutant isolates versus the WT parental strain showed that, in vitro, all mutants were less competitive than the WT strain.

\section{Signaling cascade.}

The osmosensing signaling cascades from $S$. cerevisiae, Schizosaccharomyces pombe, and several other fungi involve phosphorylation of a MAPK. In N. crassa and Colletotrichum lagenarium, the corresponding MAPKs have additionally been implicated in dicarboximide or phenylpyrrol resistance. Kojima and coworkers (2004) showed that fludioxonil treatment activates MAPK phosphorylation in $C$. lagenarium, $C$. heterostrophus, and B. cinerea. In a recent study, Yoshimi and coworkers (2005) showed that class III HK positively regulate MAPK activity through phosphorylation under hyperosmotic conditions or fungicide treatment in C. heterostrophus and N. crassa. Regulation of MAPK activity by BOS1 in response to different treatments is currently under investigation in our laboratory. Detailed characterization of BOS1 signaling cascade, its components, and their relationship with each other is also under scrutiny, since our data suggest that BOS1 may be a novel virulence factor within a subset of the phytopathogenic fungi.

\section{MATERIALS AND METHODS}

Strains, media, and culture conditions.

Botrytis cinerea UWS111 and L (Tellier et al. 2004) are WT strains respectively isolated from infected grape (Vitis vinifera) in Australia and France. Standard procedures for culture and maintenance of $B$. cinerea WT strain and mutants were carried out on NY medium ( $2 \mathrm{~g} \mathrm{l}^{-1}$ of malt extract, $2 \mathrm{~g} \mathrm{l}^{-1}$ of yeast extract, $15 \mathrm{~g} \mathrm{l}^{-1}$ of agar) or PDA (Difco, Detroit) for 4 to 15 days at $21^{\circ} \mathrm{C}$ with $16 \mathrm{~h}$ of daylight per day. Evaluation of conidiation rate was estimated on 14-day-old cultures grown on NY solid medium with or without $1 \mathrm{M}$ sorbitol. Spores were harvested by adding $3 \mathrm{ml}$ of a $0.1 \%$ Tween 20 solution to the plates, followed by vigorous shaking and recovery of the conidia-enriched solution. Conidia were pelleted by centrifugation, were resuspended in $1 \mathrm{ml}$ of $0.1 \%$ Tween 20 solution, and were counted using microscopy and a Malassez chamber.

Liquid cultures were carried out in sterile Erlenmeyer flasks with Sisler medium $\left(2 \mathrm{~g} \mathrm{l}^{-1}\right.$ of yeast extract, $10 \mathrm{~g} \mathrm{l}^{-1}$ of glucose, $2 \mathrm{~g} \mathrm{l}^{-1}$ of $\mathrm{KH}_{2} \mathrm{PO}_{4}, 1.5 \mathrm{~g} \mathrm{l}^{-1}$ of $\mathrm{K}_{2} \mathrm{HPO}_{4}, 1 \mathrm{~g} \mathrm{l}^{-1}$ of $\left(\mathrm{NH}_{4}\right)_{2} \mathrm{SO}_{4}$, $0.5 \mathrm{~g} \mathrm{l}^{-1}$ of $\mathrm{MgSO}_{4} 7 \mathrm{H}_{2} \mathrm{O}$ ) inoculated with $10^{7}$ spores per milliliter for the WT strain and shaken at $150 \mathrm{rpm}$ for 16 to $24 \mathrm{~h}$. In the case of the nonsporulating mutants, $100 \mathrm{ml}$ of medium was inoculated with 10 mycelial plugs of $5-\mathrm{mm}$ diameter and was 
incubated without shaking for $24 \mathrm{~h}$. Mycelial plugs were ground using an Ultra-Turrax (IKA, Staufen, Germany) and placed into fresh medium at a total volume of $200 \mathrm{ml}$ and was incubated for an additional 24 to $48 \mathrm{~h}$ at $23^{\circ} \mathrm{C}$ and $150 \mathrm{rpm}$.

Screening for dicarboximide-resistant transformants was carried out by estimating the growth on PDA plates containing $2 \mu \mathrm{g}$ iprodione per milliliter (Bayer Cropscience, Monheim am Rhein, Germany) $120 \mathrm{~h}$ after inoculation. Further growth tests were carried out on NY medium plates supplemented with: iprodione, $25 \mu \mathrm{g} \mathrm{ml}^{-1}$; vinclozoline, $25 \mu \mathrm{g} \mathrm{ml}^{-1}$ (BASF, Limburgerhof, Germany); procymidone, $25 \mu \mathrm{g} \mathrm{ml} \mathrm{m}^{-1}$ (Sumitomo, Osaka, Japan); quintozene, $25 \mu \mathrm{g} \mathrm{ml}^{-1}$ (Hoechst, Amsterdam); fludioxonil, $1 \mu \mathrm{g} \mathrm{ml}^{-1}$ (Syngenta, Wilmington, DE, U.S.A.); hydrogen peroxide, $24 \mathrm{mM}, 36 \mathrm{mM}$; menadione, $250 \mu \mathrm{M}, 500$ $\mu \mathrm{M}$ (Sigma-Aldrich, Saint Quentin, Fallavier, France); sodium chloride, 0.3 M, 0.6 M; cadmium sulfate, $20 \mu \mathrm{M}$; copper sulfate, $6 \mathrm{mM}$; zinc chloride, $3 \mathrm{mM}$; paraquat, 1 to $10 \mathrm{mM}$; sodium dodecyl sulfate, $0.05 \%$; caffeine, $8 \mathrm{mM}$; Congo Red, 0.6 $\mathrm{mg} \mathrm{ml}^{-1}$; calcofluor white, $0.4 \mathrm{mg} \mathrm{ml}^{-1}$; polyoxin $\mathrm{B}$ or $\mathrm{D}, 40$ ng ml $\mathrm{m}^{-1}$; glucose, $0.05,0.5 \mathrm{M}$; or sorbitol, $0.05,0.5 \mathrm{M}$. These plates were inoculated with nonsporulating mycelial plugs from 4-day-old cultures on NY medium and were incubated at $21^{\circ} \mathrm{C}$ for 2 to 3 days.

\section{Southern blot analysis.}

Genomic DNA was extracted from fungal mycelium using a Sarcosyl-based protocol (Dellaporta et al. 1983). Gel electrophoresis, restriction-enzyme digestion, Southern, and Northern experiments were performed using standard protocols (Sambrook et al. 1989). DNA probes were labeled by the random primer method, using the Q-Biogen nonaprimer kit and $20 \mu \mathrm{Ci} \alpha-{ }^{32} \mathrm{P}-\mathrm{dCTP}$ as previously described (Levis et al. 1997).

\section{Gene-replacement vector construction.}

BOS1 gene (accession number AF396827) was PCR-amplified with the primers 5'- CACATTCATCTCAACCTAAC-3' (Osm11) and 5'-TTCCCCAAAAACGCCCTTCG-3' (Osm4) and the DyNAzyme EXT polymerase (Finnzymes, Espoo, Finland). The resulting 4,747-bp fragment was cloned in pGEM-T (Promega, Charbonnières, France) to make the $\mathrm{p} B O S 1$ vector. The gene-replacement vector $\mathrm{p} B O S 1 \triangle$ was generated by using the $\mathrm{p} B O S 1$ vector and a PCR strategy described by Viaud and associates (2003). The bialaphos resistance gene $B A R$ from pCB1265 (Sweigard et al. 1997) was introduced into this plasmid at the Eam1104I restriction sites created using Seamless cloning kit (Stratagene, La Jolla, CA, U.S.A.). In order to remove the middle part of BOS1 gene from the pBOS1 vector, a first PCR amplification was carried out using primers 5'-AGTTACTCTTCACACATTTTCGACACTTTCCAACAA3' (OsmA) and 5'-AGTTACTCTTCATGGTGCTTCTTCAGT TCCCTTTCG-3' (OsmB), which both contain one Eam1104I site. A second PCR with primers 5'-TTACTCTTCACCACCT GAATGGCGAATGGAAAT-3' (bar-sean-up) and 5'-TTACTCT TCAGTGCACGGAAATGTTGAATACTC-3' (bar-sean-low), which both contain one Eam1104I, site allowed the amplification of the $B A R$ gene. Both PCR products were digested by Eam1104I and ligated together. In the resulting plasmid, $\mathrm{p} B O S 1 \triangle$, the BAR gene replaces nucleotides 576 to 3,026 with respect to the start codon of the BOS1 gene, leaving 757 and 1,539 bp up- and downstream gene fragments (Fig. 1).

\section{Cloning of full-length $\mathrm{BOS1}$ gene and complementation vector construction.}

For complementation analysis, we amplified the promoter sequence of BOS1 from genomic DNA of the L strain, using the Advantage genome walker kit (Clontech. Palo Alto, CA, U.S.A.) with the gene-specific primers 5'-ACGGCATAGACG
TGGACCATTGGATTA-3' (daf5' -1 ) and 5'-TACCCGCGGCA TTTGTAAGCAGTCACCTTCTAGC-3' (daf5' Apa). The 1.3$\mathrm{kb}$ amplified fragment was cloned after ApaI-SmaI digestion into the pBluescript $\mathrm{KS}+$ vector (Stratagene) digested with the same enzymes, leading to plasmid DL17. Double-strand sequence of DL17 lead to 968-bp 5' extension of the available BOS1 sequence of the same strain (updated accession number AF396827). For functional complementation of the null mutants, we amplified the complete gene between $-1,162$ and $+4,547$ with respect to the published start codon (Cui et al. 2002), using primers 5'-CCCCTGTGATTAAACCGAGT-3' (BOS1-prom1) and 5'-AGCGGTAAGACTTTTGTCC3' (BOSterm1) and genomic DNA of the L strain as a template, using the Tth DNA-polymerase (Clontech). The amplified fragment was cloned into pGEM-T vector (New England Biolabs, Beverly, MA, U.S.A.), leading to pGEM-daf. The 5.7-kb ApaI-PstI fragment harboring the BOS1 gene was subcloned into the pBS KS+ vector (Stratagene) together with the KpnI-digested fragment containing the hygromycin-resistance gene amplified with oligonucleotide pHYG 5'CGCGGTACCGAATTCGTCG ACGTTAAC3', using plasmid pCB1003 (Sweigard et al. 1997) as a template. The resulting plasmid pSF06 was used for transformation of the $\Delta$ bos 1 mutants.

\section{B. cinerea transformation.}

Protoplasts were prepared and transformed as described previously (Levis et al. 1997). DNA-mediated transformation was carried out using $2 \mu \mathrm{g}$ of linearized $\mathrm{pBOS} 1 \triangle$ or $5 \mu \mathrm{g}$ of circular pSF06. Transformed protoplasts were plated in molten osmotically stabilized medium agar containing $100 \mu \mathrm{g}$ of bialaphos per milliliter (ammonium glufosinate; Dr. Ehrendorfer, Augsburg, Germany) or $50 \mu \mathrm{g}$ of hygromycin B per milliliter (Sigma-Aldrich). Transformants were picked after 6 to 8 days of selection-plate growth at $21^{\circ} \mathrm{C}$. Primary transformants were then subcultured twice on selective media and were then subjected to a single-spore isolation that yielded the strains used for further experimentation. PCR screening for gene-inactivation events was performed by using one primer located upstream of BOS1 gene (Osm18 5'-CCTCGATTCAAGAGCGT CAT- $3^{\prime}$ ) and one primer located inside the BAR gene (Bar547 5'-CATGCGCACGCTCGGGTCGTT-3').

\section{Pathogenicity assays.}

The infection of bean (Vicia fabae) and tomato (Lycopersicum esculentum) by $B$. cinerea was studied by inoculating detached leaves with young unsporulating mycelium grown on NY medium prior to incubation in moist chambers. Bean plants (cv. Capitol) and tomato plants (cv. Moneymaker) were grown under glasshouse conditions. Leaves were harvested from 3- to 4-week-old plants and were placed in a transparent plastic box lined with tissue moistened with sterile water. Leaves were inoculated with 5-mm diameter plugs of 4-dayold mycelium (Weeds et al. 1999). For pathogenicity tests on wounded leaves, wounds were made with a needle tip just before inoculation. Storage boxes containing inoculated leaves were incubated in a growth cabinet at $20^{\circ} \mathrm{C}$ with $16 \mathrm{~h}$ of daylight. Disease development was recorded daily as radial spread from the inoculation point to the lesion margin. Pathogenicity assays were repeated three times, using at least five leaves per assay.

Individual grape berries ( $V$. vinifera $L$. cv. Shiraz and $V$. vinifera L. cv. Isabella) were detached manually from their bunch (and their stem) at different stages of ripening. They were placed on an open and cylindrical slot in a closed 50-ml clear plastic container to create a humid environment and were orientated such that the open-stem end faced upward. The wound was filled with a $2 \times 2-\mathrm{mm}$ plug of $B$. cinerea grown on PDA 
agar (conditions that block sporulation). Infections were left to develop at $21^{\circ} \mathrm{C}$ under industrial white neon light, using a 16-h photoperiod. Infection of apples (cv. Golden) was carried out under similar conditions, except that cylindrical $5 \times 5$-mm agar plugs of mycelium were placed within holes of similar size made with a cork borer on the apple equatorial plane (three infections per apple, mycelium facing outward). Pathogenicity assays were repeated five times, each time using three fruits per assay.

\section{Microscopic examinations.}

Infection-related morphogenesis was observed on onion ( $\mathrm{Al}$ lium cepa) epidermis as previously described (Gourgues et al. 2004; Viaud et al. 2003). Conidia collected from 12-day-old cultures on NY medium supplemented with $1 \mathrm{M}$ sorbitol were resuspended in water to a final concentration of $2 \times 10^{4}$ conidia per milliliter, and droplets $(20 \mu \mathrm{l})$ of this suspension were deposited onto the hydrophobic side of the epidermis. After $18 \mathrm{~h}$ of incubation in a humid environment at $21^{\circ} \mathrm{C}$, fungal cells were stained with Coton blue and were observed using light microscopy. These assays were carried out three times with three epidermis. For each replicate, 100 conidia were examined for their ability to form infectious hyphae.

For observations of conidiophores, the aerial hyphae were detached from 1-week-old mycelium, using transparent adhesive tape that was then placed onto a microscope slide and viewed using a light-transmission microscope.

\section{ACKNOWLEDGMENTS}

This work was supported by the University of Western Sydney internal grant scheme (Australia) and by the Santé des Plantes et Environnement Department (Institut National de la Recherche Agronomique, France). We are very grateful to A. Foster (University of Kaiserslautern, Germany) for his critical reading of the manuscript.

\section{LITERATURE CITED}

Alex, L. A., Borkovich, K. A., and Simon, M. I. 1996. Hyphal development in Neurospora crassa: Involvement of a two-component histidine kinase. Proc. Natl. Acad. Sci. U.S.A 93:3416-3421.

Alonso-Monge, R., Real, E., Wojda, I., Bebelman, J. P., Mager, W. H., and Siderius, M. 2001. Hyperosmotic stress response and regulation of cell wall integrity in Saccharomyces cerevisiae share common functional aspects. Mol. Microbiol. 41:717-730.

Avenot, H., Simoneau, P., Iacomi-Vasilescu, B., and Bataille-Simoneau, N. 2005. Characterization of mutations in the two-component histidine kinase gene AbNIK1 from Alternaria brassicicola that confer high dicarboximide and phenylpyrrole resistance. Curr. Genet. 47:234-243.

Catlett, N. L., Yoder, O. C., and Turgeon, B. G. 2003. Whole-genome analysis of two-component signal transduction genes in fungal pathogens. Eukaryot. Cell 2:1151-1161.

Chang, C., and Stewart, R. C. 1998. The two-component system. Regulation of diverse signaling pathways in prokaryotes and eukaryotes. Plant Physiol. 117:723-731.

Choi, G. J., Lee, H. J., and Cho, K. Y. 1996. Lipid peroxidation and membrane disruption by vinclozolin in dicarboximide-susceptible and -resistant isolates of Botrytis cinerea. Pest Biochem. Physiol. 55:29-39.

Choi, G. J., Lee, H. J., and Cho, K. Y. 1997. Involvement of catalase and superoxide dismutase in resistance of Botrytis cinerea to dicarboximide fungicide vinclozolin. Pest Biochem. Physiol. 59:1-10.

Cui, W., Beever, R. E., Parkes, S. L., Weeds, P. L., and Templeton, M. D. 2002. An osmosensing histidine kinase mediates dicarboximide fungicide resistance in Botryotinia fuckeliana (Botrytis cinerea). Fungal Genet. Biol. 36:187-198.

Cullen, P. J., Schultz, J., Horecka, J., Stevenson, B. J., Jigami, Y., and Sprague, G. F. 2000. Defects in protein glycosylation cause SHO1dependent activation of a STE12 signaling pathway in yeast. Genetics 155:1005-1018.

de Jong, J. C., McCormack, B. J., Smirnoff, N., and Talbot, N. J. 1997. Glycerol generates turgor in rice blast. Nature 389:244-245.

Dellaporta, S. L., Wood, J., and Hicks, J. B. 1983. A plant DNA minipreparation: Version2. Plant Mol. Biol. Rep. 1:19-21.

Di Monte, D., Bellomo, G., Thor, H., Nicotera, P., and Orrenius, S. 1984a.
Menadione-induced cytotoxicity is associated with protein thiol oxidation and alteration in intracellular $\mathrm{Ca}^{2+}$ homeostasis. Arch. Biochem. Biophys. 235:343-350.

Di Monte, D., Ross, D., Bellomo, G., Eklow, L., and Orrenius, S. 1984b. Alterations in intracellular thiol homeostasis during the metabolism of enadione by isolated rat hepatocytes. Arch. Biochem. Biophys. 235:334-342.

Dixon, K. P., Xu, J. R., Smirnoff, N., and Talbot, N. J. 1999. Independent signaling pathways regulate cellular turgor during hyperosmotic stress and appressorium-mediated plant infection by Magnaporthe grisea. Plant Cell 11:2045-2058.

Elad, Y., Williamson, B., Tudzynski, P., and Delen, N. 2004. Botrytis spp., and diseases they cause in agricultural systems-An introduction. Pages 1-6 in: Botrytis: Biology, Pathology and Control. Y. Elad, B. Williamson, P. Tudzynski, and N. Delen, eds. Kluwer Academic Publishers. Dordrecht, The Netherlands.

Faretra, F., and Pollastro, S. 1991. Genetics basis of resistance to benzimidazole and dicarboximide fungicides in Botryotinia fuckeliana (Botrytis cinerea). Mycol. Res. 95:943-951.

Fujimura, M., Ochiai, N., Oshima, M., Motoyama, T., Ichiishi, A., Usami, R., Horikoshi, K., and Yamaguchi, I. 2003. Putative homologs of SSK22 MAPKK kinase and PBS2 MAPK kinase of Saccharomyces cerevisiae encoded by os-4 and os-5 genes for osmotic sensitivity and fungicide resistance in Neurospora crassa. Biosci. Biotech. Biochem. 67:186-191.

Gourgues, M., Brunet-Simon, A., Lebrun, M. H., and Levis, C. 2004. The tetraspanin BcPls1 is required for appressorium-mediated penetration of Botrytis cinerea into host plant leaves. Mol. Microbiol. 51:619-629.

Gustin, M. C., Albertyn, J., Alexander, M., and Davenport, K. 1998. MAP kinase pathways in the yeast Saccharomyces cerevisiae. Microbiol. Mol. Biol. Rev. 62:1264-1300.

Han, K. H., and Prade, R. A. 2002. Osmotic stress-coupled maintenance of polar growth in Aspergillus nidulans. Mol. Microbiol. 43:1065-1078.

Hoch, J. A. 2000. Two-component and phosphorelay signal transduction. Curr. Opin. Microbiol. 3:165-170.

Hohmann, S. 2002. Osmotic stress signaling and osmoadaptation in Yeasts. Microbiol. Mol. Biol. Rev. 66:300-372.

Howard, R. J., and Valent, B. 1996. Breaking and entering: Host penetration by the fungal rice blast pathogen Magnaporthe grisea. Ann. Rev. Microbiol. 50:491-512.

Kawasaki, L., Sanchez, O., Shiozaki, K., and Aguirre, J. 2002. SakA MAP kinase is involved in stress signal transduction, sexual development and spore viability in Aspergillus nidulans. Mol. Microbiol. 45:1153-1163.

Kojima, K., Takano, Y., Yoshimi, A., Tanaka, C., Kikuchi, T., and Okuno, T. 2004. Fungicide activity through activation of a fungal signaling pathway. Mol. Microbiol. 53:1785-1796.

Krantz, M., Becit, E., and Hohmann, S. 2006a. Comparative genomics of the HOG-signaling system in fungi. Curr. Genet. 49:137-151.

Krantz, M., Becit, E., and Hohmann, S. 2006b. Comparative genomics of the HOG pathway proteins to generate hypotheses for functional analysis. Curr. Genet. 49:152-165.

Lee, B. N., and Elion, E. A. 1999. The MAPKKK Ste11 regulates vegetative growth through a kinase cascade of shared signaling components. Proc. Natl. Acad. Sci. U.S.A. 96:12679-12684.

Leroux, P. 2004. Chemical control of Botrytis and its resistance to chemical fungicides. Pages 195-222 in: Botrytis: Biology, Pathology and Control. Y. Elad, B. Williamson, P. Tudzynski and N. Delen, eds. Kluwer Academic Publishers. Dordrecht, The Netherlands.

Leroux, P., and Fritz, R. 1984. Antifungal activity of dicarboximides and aromatic hydrocarbons and resistance to these fungicides. Pages $207-$ 237 in: Mode of Action of Antifungal Compounds. A. P. J. Trinci and J. F. S. Ryley, eds. University Press. Cambridge.

Leroux, P., Fritz, R., Debieu, D., Albertini, C., Lanen, C., Bach, J., Gredt, M., and Chapeland, F. 2002. Mechanisms of resistance to fungicides in field strains of Botrytis cinerea. Pest Manag. Sci. 58:876-888.

Levis, C., Fortini, D., and Brygoo, Y. 1997. Transformation of Botrytis cinerea with the nitrate reductase gene (niaD) shows a high frequency of homologous recombination. Curr. Genet. 32:157-162.

Mac Clellan, W. D., and Hewitt, W. B. 1973. Early Botrytis rot of grapes: Time of infection and latency of Botrytis cinerea Pers. in Vitis vinifera. Phytopathology 63:1151-1157.

Maeda, T., Takekawa, M., and Saito, H. 1995. Activation of the yeast PBS2 MAPKK by MAPKKKs or by binding of an SH3-containing osmosensor. Science 269:554-558.

Motoyama, T., Kadokura, K., Ohira, T., Ichiishi, A., Fujimura, M. Yamaguchi, I., and Kudo, T. 2005a. A two-component histidine kinase of the rice blast fungus is involved in osmotic stress response and fungicide action. Fungal Genet. Biol. 42:200-212.

Motoyama, T., Ohira, T., Kadokura, K., Ichiishi, A., Fujimura, M., Yamaguchi, I., and Kudo, T. 2005b. An Os-1 family histidine kinase 
from a filamentous fungus confers fungicide-sensitivity to yeast. Curr. Genet. 47:298-306

Moyano, C., Gomez, V., and Melgarejo, P. 2004. Resistance to pyrimethanil and other fungicides in Botrytis cinerea populations collected on vegetable crops in Spain. J. Phytopathol. 152:484-490.

Ochiai, N., Fujimura, M., Motoyama, T., Ichiishi, A., Usami, R., Horikoshi, K., and Yamaguchi, I. 2001. Characterization of mutations in the twocomponent histidine kinase gene that confer fludioxonil resistance and osmotic sensitivity in the os-1 mutants of Neurospora crassa. Pest Manag. Sci. 57:437-442.

O'Rourke, S. M., and Herskowitz, I. 1998. The Hog1 MAPK prevents cross talk between the HOG and pheromone response MAPK pathways in Saccharomyces cerevisiae. Genes Dev. 12:2874-2886.

O'Rourke, S. M., Herskowitz, I and O'Shea, E. K. 2002. Yeast go the whole HOG for the hyperosmotic response. Trends Genet. 18:405-12.

Park, G., Bruno, K. S., Staiger, C. J., Talbot, N. J., and Xu, J. R. 2004. Independent genetic mechanisms mediate turgor generation and penetration peg formation during plant infection in the rice blast fungus. Mol. Microbiol. 53:1695-1707.

Raposo, R., Gomez, V., Urrutia, T., and Melgarejo, P. 2000. Fitness of Botrytis cinerea associated with dicarboximide resistance. Phytopathol. 90:1246-1249.

Sambrook, J., Fritsch, E. F., and Maniatis, T. 1989. Molecular Cloning: A Laboratory Manual. Cold Spring Harbor Laboratory Press, Cold Spring Harbor, NY, U.S.A.

Schumacher, M. M., Enderlin, C. S., and Selitrennikoff, C. P. 1997. The osmotic-1 locus of Neurospora crassa encodes a putative histidine kinase similar to osmosensors of bacteria and yeast. Curr. Microbiol. 34:340-347

Sweigard, J., Chumley, F., Caroll, A., Farrall, L., and Valent, B. 1997. A series of vectors for fungal transformation. Fungal Genet. Newsl. 44:52-53.

Tellier, F., Carlin-Sinclair, A., Fritz, R., Cherton, J. C., and Leroux, P. 2004. Activity and metabolism of cyano-oxime derivatives in various strains of Botrytis cinerea. Pest. Biochem. Physiol. 78:151-160.

Tzeng, W. F., Lee, J. L., and Chiou, T. J. 1995. The role of lipid-peroxidation in menadione-mediated toxicity in cardiomyocytes. J. Mol. Cell. Cardiol. 27:1999-2008.

Van Wuytswinkel, O., Reiser, V., Siderius, M., Kelders, M. C., Ammerer, G., Ruis, H., and Mager, W. H. 2000. Response of Saccharomyces cerevisiae to severe osmotic stress: Evidence for a novel activation mechanism of the HOG MAP kinase pathway. Mol. Microbiol. 37:382-397.

Viaud, M., Brunet-Simon, A., Brygoo, Y., Pradier, J. M., and Levis, C. 2003. Cyclophilin A and calcineurin functions investigated by gene inactivation, cyclosporin A inhibition and cDNA arrays approaches in the phytopathogenic fungus Botrytis cinerea. Mol. Microbiol. 50:14511465 .

Virginia, M., Appleyard, C. L., McPheat, W. L., and Stark, M. J. 2000. A novel 'two-component' protein containing histidine kinase and response regulator domains required for sporulation in Aspergillus nidulans. Curr. Genet. 37:364-372.

Weeds, P. L., Beever, R. E., Sharrock, K. R., and Long, P. G. 1999. A major gene controlling pathogenicity in Botryotinia fuckeliana (Botrytis cinerea). Physiol. Mol. Plant Pathol. 54:13-35.

West, A. H., and Stock, A. M. 2001. Histidine kinases and response regulator proteins in two-component signaling systems. Trends Biochem. Sci. 26:369-376.

Wolanin, P. M., Webre, D. J., and Stock, J. B. 2003. Mechanism of phosphatase activity in the chemotaxis response regulator CheY. Biochemistry 42:14075-14082.

Yoshimi, A., Tsuda, M., and Tanaka, C. 2004. Cloning and characterization of the histidine kinase gene Dic1 from Cochliobolus heterostrophus that confers dicarboximide resistance and osmotic adaptation. Mol. Genet. Genomics 271:228-236.

Yoshimi, A., Kojima, K., Takano, Y., and Tanaka, C. 2005. Group III histidine kinase is a positive regulator of Hog1-type mitogen-activated protein kinase in filamentous fungi. Eukaryot. Cell 4:1820-1828.

Zhang, Y., Lamm, R., Pillonel, C., Lam, S., and Xu, J. R. 2002. Osmoregulation and fungicide resistance: The Neurospora crassa os-2 gene encodes a HOG1 mitogen-activated protein kinase homologue. Appl. Environ. Microbiol. 68:532-538.

Ziogas, B. N., Markoglou, A. N., and Spyropoulou, V. 2005. Effect of phenylpyrrole-resistance mutations on ecological fitness of Botrytis cinerea and their genetical basis in Ustilago maydis. Eur. J. Plant Pathol. 113:83-100.

\section{AUTHOR-RECOMMENDED INTERNET RESOURCE}

Broad Institute Botrytis cinerea database: www.broad.mit.edu/annotation/genome/botrytis_cinerea/Home.html 